\title{
BMJ Open Effectiveness of a hydrogel dressing as an analgesic adjunct to first aid for the treatment of acute paediatric burn injuries: a prospective randomised controlled trial
}

\author{
Maleea Denise Holbert (D) , ${ }^{1,2,3}$ Roy M Kimble, ${ }^{1,2,3,4}$ Mark Chatfield, ${ }^{3}$ \\ Bronwyn R Griffin ${ }^{1,2,4,5}$
}

To cite: Holbert MD, Kimble RM, Chatfield $\mathrm{M}$, et al. Effectiveness of a hydrogel dressing as an analgesic adjunct to first aid for the treatment of acute paediatric burn injuries: a prospective randomised controlled trial. BMJ Open 2021;11:e039981. doi:10.1136/ bmjopen-2020-039981

- Prepublication history and additional material for this paper is available online. To view these files, please visit the journal online (http://dx.doi.org/10. 1136/bmjopen-2020-039981)

Received 01 May 2020

Revised 16 December 2020

Accepted 16 December 2020

Check for updates

(C) Author(s) (or their employer(s)) 2021. Re-use permitted under CC BY-NC. No commercial re-use. See rights and permissions. Published by BMJ.

For numbered affiliations see end of article.

Correspondence to Ms Maleea Denise Holbert; m.holbert@uq.edu.au

\section{ABSTRACT}

Objective To compare the effectiveness of two acute burn dressings, Burnaid hydrogel dressing and plasticised polyvinylchloride film, on reducing acute pain scores in paediatric burn patients following appropriate first aid. Design Single-centre, superiority, two-arm, parallelgroup, prospective randomised controlled trial. Participants and setting Paediatric patients (aged s16) presenting to the Emergency Department at the Queensland Children's Hospital, Brisbane, Australia, with an acute thermal burn were approached for participation in the trial from September 2017-September 2018.

Interventions Patients were randomised to receive either (1) Burnaid hydrogel dressing (intervention) or (2) plasticised polyvinylchloride film (Control) as an acute burn dressing.

Primary and secondary outcomes Observational pain scores from nursing staff assessed 5 min post application of the randomised dressing, measured using the Face Legs Activity Cry and Consolability Scale was the primary outcome. Repeated measures of pain, stress and re-epithelialisation were also collected at follow-up dressing changes until $95 \%$ wound re-epithelialisation occurred.

Results Seventy-two children were recruited and randomised ( $n=37$ intervention; $n=35$ control). No significant between-group differences in nursing (mean difference: $-0.1,95 \% \mathrm{Cl}-0.7$ to $0.5, \mathrm{p}=0.72$ ) or caregiver (MD: $1,95 \% \mathrm{Cl}-8$ to $11, \mathrm{p}=0.78$ ) observational pain scores were identified. Moreover, no significant differences in child self-report pain (MD: $0.3,95 \% \mathrm{Cl}$ -1.7 to $2.2, \mathrm{p}=0.78$ ), heart rate (MD: $-3,95 \% \mathrm{Cl}-11$ to $5, \mathrm{p}=0.41$ ), temperature (MD: $0.6,95 \% \mathrm{Cl}-0.13$ to 0.24 , $\mathrm{p}=0.53$ ), stress (geometric mean ratio: $1.53,95 \% \mathrm{Cl} 0.93$ to $2.53, p=0.10$ ), or re-epithelialisation rates (MD: -1 , $95 \% \mathrm{Cl}-3$ to $1, p=0.26$ ) were identified between the two groups.

Conclusions A clear benefit of Burnaid hydrogel dressing as an analgesic adjunct to first aid for the treatment of acute paediatric burns was not identified in this investigation.

Trial registration number Australian New Zealand Clinical Trials Registry (ACTRN12617001274369).
Strengths and limitations of this study

- First randomised controlled trial investigating analgesic properties of acute burn dressings in a paediatric burn population.

- Pain was assessed using age-specific and reliable self-report and observational scales, in addition to physiological measures of pain and distress.

- This investigation was pragmatic in nature, replicating real-world clinical scenarios where acute burn dressings are used.

- Lack of representativeness within the patient sample (small to medium sized burns in children aged between 0 and 5 years) may limit generalisability of the findings to the broader paediatric burn population.

\section{INTRODUCTION}

Pain remains a major issue following a burn, and research suggests that pain from burn injuries continues to be undertreated in children. ${ }^{1}$ The subsequent wound care required to treat a burn is also associated with significant pain and distress-thus burn pain comprises a challenging spectrum of acute, background, breakthrough and procedural pain. ${ }^{23}$ The aim of this trial was to provide health practitioners with evidence to support the use of an acute burn dressing that is superior in terms of pain relief for paediatric patients with acute thermal burn injuries. Optimising pain management for paediatric burn patients is more than just a compassionate need to reduce suffering-despite that being a sufficient motivator for healthcare professionals. Improving acute pain control for children with traumatic injuries such as a burn is critical, as suboptimal analgesia can prolong wound re-epithelialisation. ${ }^{45}$ Moreover, adverse and uncontrolled 
pain can have long-term emotional consequences ${ }^{67}$ and influence pain perception and processing later in life. ${ }^{89}$

Topical administration of cool running water (CRW) for 20 min within 3 hours of the burn occurring is the recommended gold standard first aid for burn injuries, in accordance with the Australian and New Zealand Burn Association. ${ }^{10-14}$ Following first aid, guidelines recommend burn wounds to be covered with a sterile dressing to maintain a moist wound environment, minimise the risk of infection, and prevent air exposure-as this can be quite painful for patients with acute burns. ${ }^{15}$ Characteristics of an ideal acute burn dressing include a transparent non-adherent design, easy application and removal, and protection from environmental exposure. Plasticised polyvinylchloride (PVC) film fulfils this criteria, and excluding the application to facial burns, is an inexpensive and practical dressing for acute burn injuries in the prehospital and emergency department (ED) setting. For this reason, PVC film has been used in the management of acute burns for over four decades. However, the preferred acute burn dressing varies between prehospital services in different states and countries.

Over the past decade, Burnaid hydrogel dressings have gained widespread use in the prehospital setting for acute burn injuries-and are promoted as providing hydration to the burn wound and pain relief via a convection and evaporative cooling effect. ${ }^{16}$ Burnaid dressings comprise of a $3 \mathrm{~mm}$ thick sterile polyester urethane foam pad impregnated with a propylene glycol gel, which contains more than $90 \%$ purified water. Despite its popularity among prehospital services, there is limited empirical evidence for the effectiveness of hydrogel burn dressings, and no studies have been conducted in a paediatric burn population. At present, there is no robust empirical evidence to support the adoption of one particular acute burn dressing over the others. With the continual development of expensive wound care products, it is important that we validate their use and effectiveness within the targeted clinical population. This trial examined the effectiveness of Burnaid hydrogel dressings as an analgesic adjunct to first aid for the treatment of acute paediatric burns in comparison to current standard practice-PVC film. While PVC film offers protection from the external environment, Burnaid dressings provide evaporative cooling and a significant reduction in subdermal temperatures when air currents pass over the dressing. ${ }^{17}$ This evaporative cooling effect, which is specific to hydrogel dressings, was the expected benefit of Burnaid in comparison to PVC film. This evaporative cooling effect was also why Burnaid dressings were hypothesised to provide superior pain relief compared with the current standard acute burn dressing.

\section{METHODS}

\section{Design and setting}

We conducted a prospective, single-centre, superiority, randomised controlled trial (RCT) examining the effectiveness of a hydrogel dressing as an analgesic adjunct to first aid for the treatment of acute paediatric burn injuries, compared with current standard care. We used a two-arm parallel design with a 1:1 allocation ratio. Participants were recruited between September 2017 and September 2018 from the ED and the Pegg Leditschke Children's Burns Outpatient Department (OPD) at the Queensland Children's Hospital (QCH) following initial presentation for their burn. The QCH serves as the major burns referral centre for Queensland and Northern New South Wales, treating over 1200 paediatric patients with burn injuries per annum.

\section{Patient and public involvement}

Patients and/or the public were not involved in the development of this research. However, relevant stakeholders and knowledge users (ie, prehospital staff, clinicians, and nurses) were involved in the initial development of the trial, refinement of research questions and identification of current knowledge gaps.

\section{Protocol and registration}

Study methodology was documented in a published protocol $^{18}$ and registered with the Australian New Zealand Clinical Trials Registry (ID number: ACTRN12617001274369) on the 5 September 2017 prior to recruitment. This trial was completed as per the published protocol, ${ }^{18}$ which contains a more in-depth description of the trial's design and methods.

\section{Participants}

\section{Inclusion criteria}

Inclusion criteria: children aged between 0 and 16 years with an acute thermal burn $<20 \%$ of the child's total body surface area (TBSA), presented to the ED or Burns OPD within 24 hours of sustaining the burn, received optimal first aid, and no definitive silver dressings or silver sulphadiazine cream applied prior to enrolment.

\section{Exclusion criteria}

Exclusion criteria included: children with non-thermal burns or inhalation injuries, presented to the QCH more than 24-hours post burn, inadequate first aid, prior treatment with silver wound products, non-English speaking, cognitive impairments, required ventilation or initial debridement under general anaesthetic, current involvement with Department of Communities, known sensitivity to hydrogels, and patients with comorbidities that could impair wound healing or exacerbate/alter pain (ie, metabolic congenital disorders, spinal cord defects/injuries, insensate patients).

\section{Procedures}

Participant enrolment and intervention allocation are described in figure 1. All participants (if age appropriate) and caregivers were given verbal and written information about the research, and provided signed consent to participate in the trial. After obtaining informed consent, participants were stratified by pain risk (1. High Pain or 2 . 


\section{Enrolment}
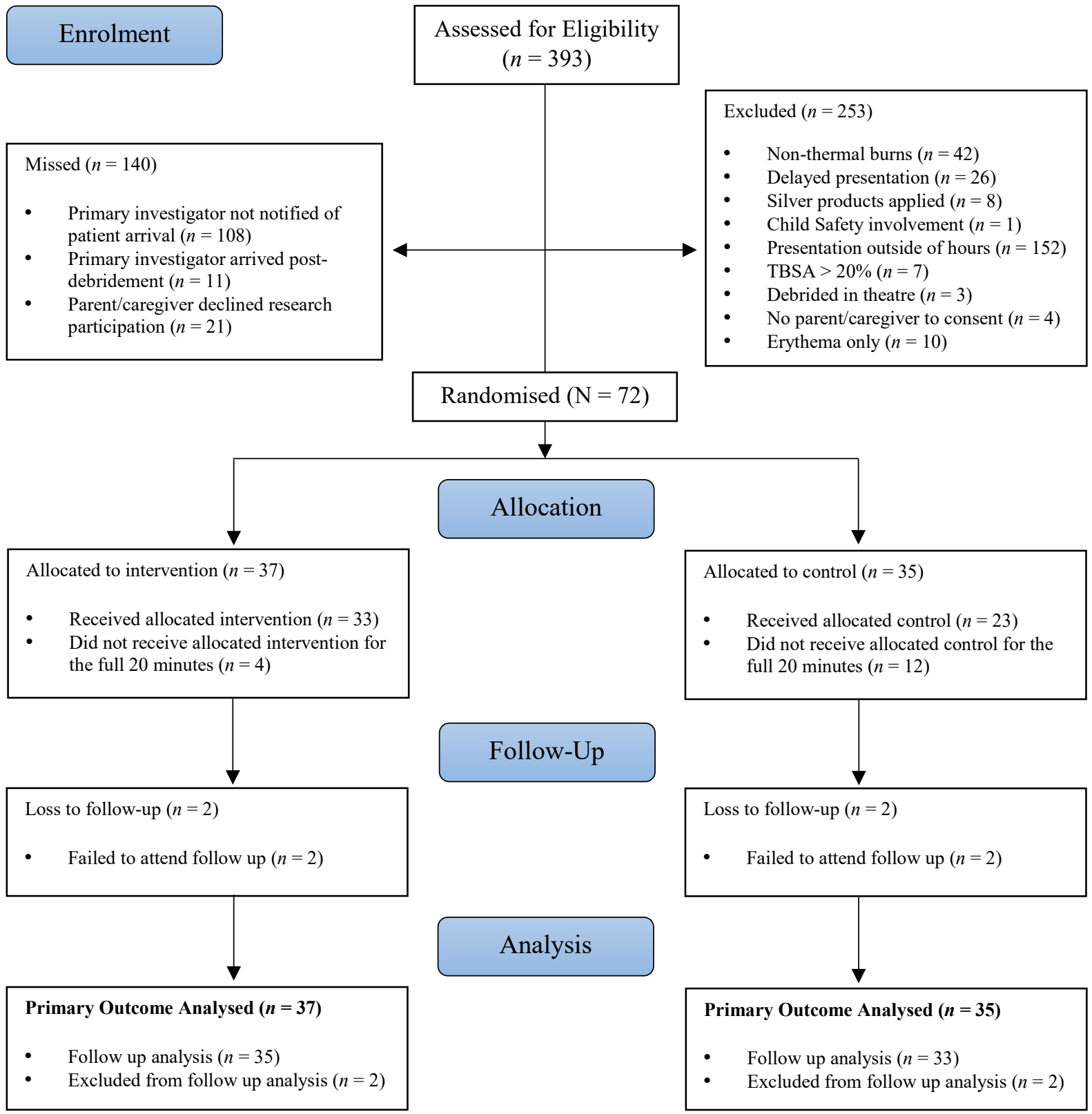

Figure 1 Consolidated Standards of Reporting Trials flow diagram. TBSA, total body surface area.

Low Pain) according to factors that could influence pain in paediatric burn patients. Factors were based on findings from a retrospective review of data from the Queensland Paediatric Burns Registry (unpublished hospital quality review). Participants presenting to the ED or Burns OPD with one or more of the following criteria were considered at high pain risk: unilateral or bilateral foot burns, campfire/hot coal burns, circumferential burn injuries, and burns $>5 \%$ TBSA. Following stratification, patients were randomised to receive either (1) Burnaid hydrogel dressing (Intervention) or (2) PVC film (Control). A computerised random number sequence-generating programme was used for participant randomisation. Concealment of treatment allocation were performed via the use of sealed, opaque, identical, consecutively numbered envelopes prepared in advance by an independent third party.

Due to the pragmatic nature of this trial, researchers could not be blinded to which randomised dressing patients received. Researchers were required to be present when the acute burn dressings were applied and removed to obtain pain scores and additional outcome measures from the child, caregiver and medical staff. Treating clinicians, nursing staff, patients and caregivers were also not blinded to which treatment participants received as dressings were visible on the patient's burn. Because these dressings are topical, concealment during patient treatment in the ED was not possible. To include an element of blinding in the trial, a specialist panel of burn surgeons and senior nurses performed a blinded 


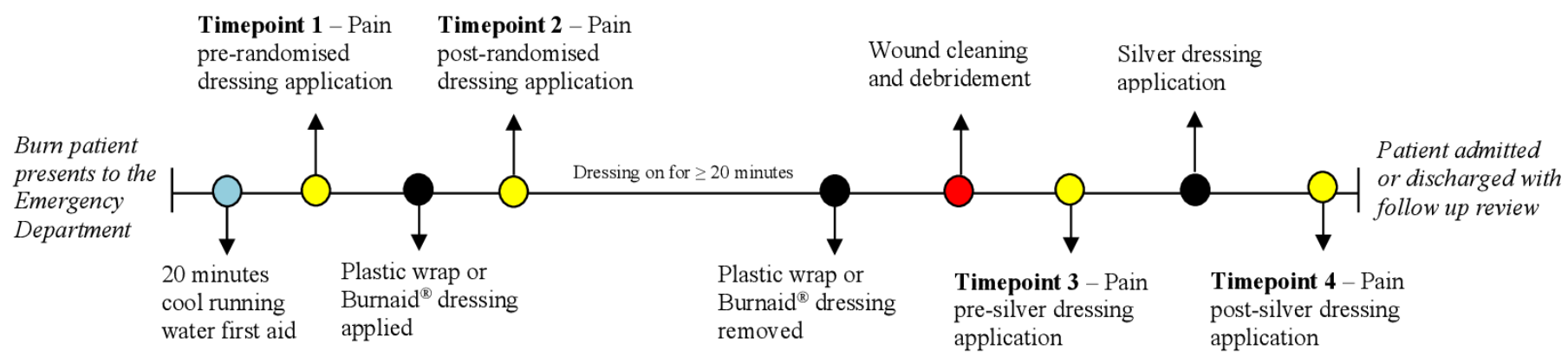

$B$

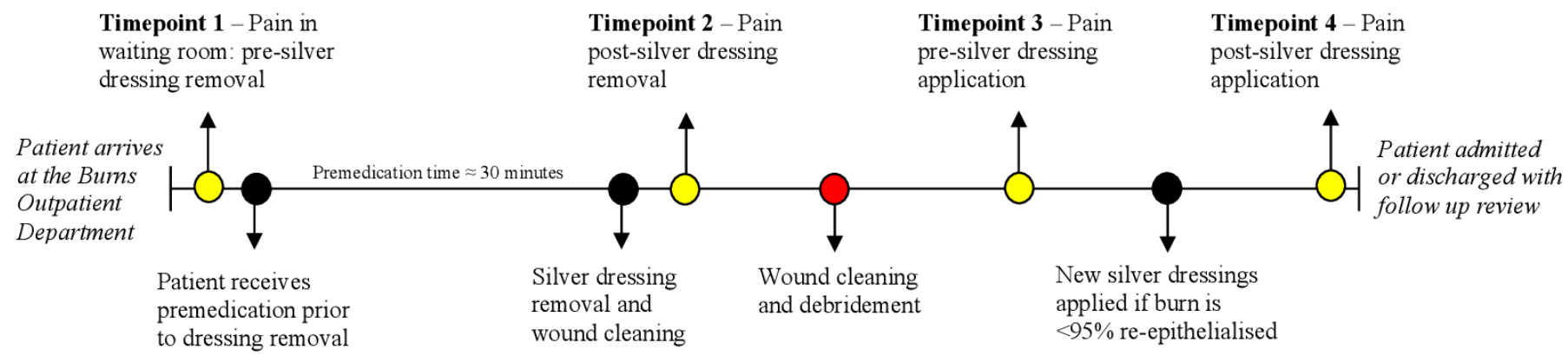

Figure 2 Pain assessment timepoints during acute and follow-up care.

review of 3D wound images to determine rate of re-epithelialisation at each dressing change until $>95 \%$ burn re-epithelialisation occurred.

Pain was assessed in the ED (figure 2A) at four timepoints relative to the child's acute treatment for their burn: (T1) prerandomised dressing application, (T2) postrandomised dressing application, (T3) predefinitive dressing application and (T4) postdefinitive dressing application. Peak pain during wound cleaning and debridement was also collected from nursing staff using the Face, Legs, Activity, Cry and Consolability (FLACC), aiming to capture the worst/maximal pain experienced during acute treatment. During subsequent dressing changes in the Burns OPD (figure 2B), pain was assessed at four time points relative to the child's follow-up treatment: (T1) predefinitive-dressing removal, (T2) postdefinitive dressing removal, (T3) predefinitive dressing application, (T4) postdefinitive dressing application. Peak pain during wound cleaning was also documented during dressing changes. Observational pain scores from ED nursing staff assessed post application of the randomised dressings (T2 in figure 2A) was the primary outcome measure of the trial. Pain at T2 was assessed 5 min after the application of the randomised dressings for all participants-to give the dressings a standard period of time on the wound before pain assessment.
Randomised dressings were left in place for $20 \mathrm{~min}$. This time duration was chosen as the standardised time for dressings to be applied in the ED for two reasons. First, this duration was predicted to be the time taken from patient presentation to surgical assessment in the ED-prior to wound debridement and definitive dressing application. This time duration was discussed with key stakeholders and relevant knowledge users (such as ED consultants, surgical consultants and nursing staff) prior to recruitment and data collection for the trial. Second, $20 \mathrm{~min}$ has previously been used as the standardised time duration for the application of Burnaid dressings in a burn porcine model. ${ }^{17}$ As little-to-no research has been conducted examining acute burn dressings in a paediatric ED setting, and Burnaid dressings do not provide a minimum duration for dressing application, 20 min was used as a standardised duration to ensure consistency between participants.

Additional measures collected at each of the eight aforementioned timepoints during the child's acute and follow-up care included: a saliva sample (to measure biomarkers of stress), heart rate and temperature. The duration of each burn care procedure was timed in the ED and Burns OPD. Information regarding analgesic medication administered to the patient prior to enrolment in the trial was obtained from Ambulance chart records and 
referral notes. All medication administered to patients following presentation to the QCH was recorded, in addition to all non-pharmacological interventions such as distraction techniques, rewards, procedural preparation and music/behavioural therapies.

\section{Interventions}

Intervention-Burnaid hydrogel dressing

Burnaid hydrogel dressing (Mundicare, Sydney, Australia) served as the treatment intervention in this trial. Burnaid products previously contained Melaleuca alternifolia (tea tree) for its broad-spectrum antimicrobial properties; however, inclusion of this active ingredient has since ceased and no tea tree containing Burnaid products were used in this investigation.

\section{Control-plasticised polyvinylchloride film}

Plasticised PVC film (also known as plastic wrap, cling film, and Saran wrap) is a thin $(<25 \mu \mathrm{m})$ food-wrap that has been used in the management of acute burn injuries for over four decades. ${ }^{19} 20$

\section{Measurements}

Primary outcome measure

Observational pain scores from ED nursing staff was the primary outcome measure of the trial, and was assessed using the FLACC scale. The FLACC scale is a five-item composite tool measuring aspects of both pain and distress in children. The scale consists of five categories of behaviour, each of which are scored on a 0 to 2 points scale, giving a total score ranging from 0 to $10 .^{21}$ The FLACC has been described in the literature as a reliable and well-validated pain assessment tool for postoperative pain in patients age between 0 and 7 , and has shown correlations with child self-report pain measures. ${ }^{22}{ }^{23}$ This pain scale was chosen to be the primary outcome measure for the trial based on the low median age range of patients presenting to the QCH with a burn. While selfreport pain measures are acknowledged to be the gold standard, a significant proportion of patients presenting to the ED for acute burn treatment are preverbal and thus unable to self-report their pain.

\section{Additional measures of pain}

\section{Child self-report (ages 4-8 years)}

Child self-report pain scores were assessed using the Faces Pain Scale-Revised (FPS-R). The FPS-R is a linear selfreport scale designed for pain assessment in children over the age of four. ${ }^{24} 25$ The item is composed of six-points (six-faces with differing expressions) with a lower anchor of no pain and an upper anchor of very much pain.

\section{Child self-report (ages 8+)}

For patients over the age of eight, self-report pain was assessed using the Visual Analogue Scale for Pain (VAS). The VAS has been described in the literature as a reliable and well-validated pain assessment tool for use in older children. ${ }^{26} 27$

\section{Parent (observational) report}

Parent/caregiver observational pain scores were assessed using the Observer Visual Analogue Scale for Pain (VAS Observer) for preverbal paediatric patients and those under the age of 4 . The VAS Observer has been shown to be a reliable and valid observational pain scale for use in a non-verbal paediatric population, and for children who are unable to self-report their pain. ${ }^{28}$

\section{Secondary outcome measures \\ Re-epithelialisation}

Burns were considered re-epithelialised if $\geq 95 \%$ of the original wound area had re-epithelialised, and the patient no longer required definitive dressings. Wound re-epithelialisation was assessed using two methods. First, clinical judgement from the treating surgical consultant was determined at each dressing change. Second, a panel of paediatric burn specialists performed a blinded review of 3D images (3D LifeViz System; QuantifiCare, Valbonne, France) of patient's burn wounds taken at each dressing change.

\section{Stress}

Stress was assessed in this trial using $\alpha$-amylase-a biochemical stress marker produced locally within salivary glands. Patients placed a SalivaBio Oral Swab (Salimetrics Europe, Newmarket, UK) under their tongue for $2 \mathrm{~min}$ for saliva collection. Salivary Alpha-Amylase Kinetic Enzyme Assay Kits (Item No. 1-1902, Salimetrics) were used to quantify $\alpha$-amylase, as per the manufacturer's instructions. The trial protocol included assessments of levels of $\alpha$-amylase and cortisol as indicators of stress during burn wound treatment in the ED and subsequent dressing changes. Salivary a-amylase (sAA) was selected over cortisol based on previous research conducted at the Pegg Leditschke Paediatric Burns OPD. ${ }^{29}$ This research found sAA to be responsive to stress during wound care procedures, and also found an association between sAA and pain in children with thermal burns during dressing changes. Moreover, follow-up appointments occur during a morning clinic which runs from 07:30 to 10:00. Cortisol levels are known to peak within 30-45 min of waking up and then decrease due to diurnal variation. Due to the timing of sample collection, sAA was deemed to be a more appropriate measure of stress in this trial. Saliva samples were analysed from the following timepoints:

1. Preapplication and postapplication of the randomised dressing (ie, Burnaid or PVC film).

2. Following patient arrival in the Burns OPD for their first dressing change-prior to premedication and definitive dressing removal.

3. Following patient arrival in the Burns OPD for their second dressing change-prior to premedication and definitive dressing removal.

\section{Staff and caregiver perspectives on dressings}

Dressing satisfaction from clinical staff regarding ease of randomised dressing application, ease of removal, 
flexibility and conformity were rated using a self-report 0-10 Numeric Rating Scale (NRS) for both Burnaid dressings and PVC film from ED nursing staff. Parent/ caregiver ratings on ease of dressing application, removal, comfort and ease of movement were also assessed using a $0-10$ NRS. It is acknowledged that ease of dressing measurements within the ED were confounded due to lack of blinding, and as a result of the variable nature, size and anatomical location of the areas to be dressed.

\section{Demographic and clinical information}

Demographic and clinical details were obtained from parents/caregivers and medical records including age, sex, burn mechanism, area affected, estimated burn TBSA, and prehospital care (such as first aid and pharmacological interventions). Treating surgical staff first assessed burn TBSA in the ED following wound debridement using a modified version of the Lund and Browder chart. ${ }^{30}$ Burn TBSA was also assessed at each change of dressing from the child's treating consultant until the burns were considered to be $95 \%$ re-epithelialised. Burn depth was assessed using two methods in the trial. First, clinical judgement from the treating surgical consultant was reported following initial patient presentation to the hospital, and at each follow-up appointment in the Burns OPD for dressing changes. Second, burn depth was assessed using rapid imaging with Moor LDLS-BI Laser Doppler Imager (Moor Instruments Limited, Devon, UK). Laser Doppler Imaging (LDI) is a non-contact technique used in the assessment of burn injuries to measure skin blood perfusion at the surface of the burn wound. ${ }^{31}$ LDI measures the extent of microvessel blood flow within the whole burn area, providing information on burn depth via microcirculation expressed as 'perfusion units' (PU). ${ }^{32}$ Participants had their burn wounds scanned using LDI on their first change of dressing (72-120 hours post burn) in the Burns Outpatient Department to obtain mean and minimum PU. This time period for LDI is in accordance with the manufactures instructions, and has been established as acceptable time frame in recent studies. ${ }^{3435}$

\section{Statistical analysis}

In accordance with previous studies aiming to reduce pain in paediatric burn patients, we expected pain scores within each treatment group to have a normal distribution and a SD of 2.4. ${ }^{36}$ Data were analysed on an intent-totreat basis. Sample size was estimated at 29 experimental (intervention) participants and 29 control participants to detect a significant between-group difference of 1.8 in pain scores postdressing application. With power equal to $0.8, \alpha$ set at 0.05 , and up to a potential $20 \%$ loss to follow-up, the calculated target sample size was 72 participants. Between-group differences in primary and secondary outcomes were estimated using mixed models in Stata V.16. ${ }^{37}$ Random effects for patients accounted for the repeated measures, and restricted maximum likelihood method with Kenward-Rogers df was used. Each model included data at baseline (ie, predressing) and at one follow-up time, and assumed no population differences at baseline, a change from baseline in the control group and a different change from baseline in the intervention group. Adjusted mean differences (interventioncontrol) and 95\% CIs are reported. The sAA data were log-transformed, and the adjusted ratio of geometric means (intervention $\div$ control) are reported..$^{38}$

\section{RESULTS}

\section{Sample and demographic characteristics}

Seventy-two paediatric burn patients were randomised and recruited into the trial. Four participants were lost to follow-up and had no additional data collected past the initial point of treatment in the ED. Patient demographic details and baseline characteristics are presented in table 1.

No adverse events occurred in the intervention or control group, and no baseline population differences were identified. Throughout data collection, no children in the 4-8 age group reported having trouble selfreporting their pain to the investigator using the FPS-R. Data were collected for dressing changes $4(n=8), 5$ $(\mathrm{n}=4), 6(\mathrm{n}=1), 7(\mathrm{n}=1), 8(\mathrm{n}=1), 9(\mathrm{n}=1)$ and $10(\mathrm{n}=1)$ for patients requiring multiple dressing changes, but were not included in the analysis due to low numbers of participants in the trial requiring more than four dressings.

Successful LDI scans were completed for 58 out of the 72 participants during their first burn dressing change. The revised standard scale of $0-1000 \mathrm{PU}$ was used to measure burn depth from LDI scans. In accordance with previous studies, 0-250 PU indicated full-thickness injuries, 250-625 PU represented deep dermal partial thickness burns, and $>625$ PU corresponded to superficial partial thickness burns. ${ }^{39}$ T-tests revealed no significant difference in LDI scores between the intervention or control group for mean perfusion, $\mathrm{p}=0.79$. In addition, no difference in minimum LDI scores were found between the intervention or control group, $p=0.20$. Mean PUs for both groups were greater than or equal to 625 PU indicating superficial partial thickness burn injuries. These values support clinical judgement from the treating surgical consultants for burn depth assessment (see table 1).

\section{Primary outcome}

Acute pain scores collected in the ED before and after the application of the randomised dressing (T1 and T2), and before and after definitive dressing application (T3 and T4), are reported in table 2 for the two groups. No significant between-group differences in pain scores (assessed using the FLACC scale from nursing staff) were found between paediatric patients who received Burnaid dressings and those who received PVC film as an acute burn dressing in the ED following initial presentation to the QCH and CRW first aid. No significant group differences in FLACC scores were found postrandomised dressing 
Table 1 Participant demographic and clinical variables

\begin{tabular}{|c|c|c|}
\hline Variable & $\begin{array}{l}\text { Intervention } \\
\mathrm{n}=37\end{array}$ & $\begin{array}{l}\text { Control } \\
n=35\end{array}$ \\
\hline \multicolumn{3}{|l|}{ Patient age (years) } \\
\hline $0-3$ & $20(54 \%)$ & $27(77 \%)$ \\
\hline $4-7$ & $9(24 \%)$ & $5(14 \%)$ \\
\hline $8-16$ & $8(22 \%)$ & $3(9 \%)$ \\
\hline \multicolumn{3}{|l|}{ Indigenous status } \\
\hline Not indigenous & $34(92 \%)$ & 33 (94\%) \\
\hline Aboriginal & $2(5 \%)$ & $2(6 \%)$ \\
\hline Torres Strait Islander & $1(3 \%)$ & $0(0 \%)$ \\
\hline \multicolumn{3}{|l|}{ Gender } \\
\hline Male & 22 (59\%) & $19(54 \%)$ \\
\hline \multicolumn{3}{|l|}{ Mechanism of injury } \\
\hline Scald & 26 (70\%) & 28 (80\%) \\
\hline Contact & $8(22 \%)$ & $7(20 \%)$ \\
\hline Flame & $2(5 \%)$ & $0(0 \%)$ \\
\hline Flash & $1(3 \%)$ & $0(0 \%)$ \\
\hline \multicolumn{3}{|l|}{ Burn source } \\
\hline Hot beverage & $10(27 \%)$ & $14(40 \%)$ \\
\hline $\begin{array}{l}\text { Water from kettle/ } \\
\text { saucepan/tap }\end{array}$ & $7(19 \%)$ & $10(29 \%)$ \\
\hline Noodles & $7(19 \%)$ & $3(9 \%)$ \\
\hline Food (other) & $1(3 \%)$ & $1(3 \%)$ \\
\hline Stove/oven/barbeque & $4(11 \%)$ & $3(9 \%)$ \\
\hline Lighter & $2(5 \%)$ & $0(0 \%)$ \\
\hline $\begin{array}{l}\text { Hair straightener/curling } \\
\text { iron }\end{array}$ & $1(3 \%)$ & $2(6 \%)$ \\
\hline Fireplace/sun heated metal & $2(5 \%)$ & $2(6 \%)$ \\
\hline Hot oil/wax & $2(5 \%)$ & $0(0 \%)$ \\
\hline Aerosol can explosion & $1(3 \%)$ & $0(0 \%)$ \\
\hline Burn TBSA percentage & $2(1-4)$ & $2(1-4)$ \\
\hline \multicolumn{3}{|l|}{ Burn depth } \\
\hline Superficial partial thickness & $30(81 \%)$ & $24(69 \%)$ \\
\hline $\begin{array}{l}\text { Deep dermal partial } \\
\text { thickness }\end{array}$ & $7(19 \%)$ & $11(31 \%)$ \\
\hline Burn wound perfusion & $n=48^{*}$ & $n=43^{*}$ \\
\hline LDI mean PU & $696(293)$ & $679(276)$ \\
\hline LDI Minimum PU & $144(143)$ & 110 (104) \\
\hline \multicolumn{3}{|l|}{ Anatomical region affected } \\
\hline Upper limb and/or hand & $19(51 \%)$ & $20(57 \%)$ \\
\hline Lower limb and/or foot & $11(30 \%)$ & $10(29 \%)$ \\
\hline $\begin{array}{l}\text { Chest, abdomen, and/or } \\
\text { back }\end{array}$ & $12(32 \%)$ & $13(37 \%)$ \\
\hline Head, face, and/or neck & $8(22 \%)$ & $10(29 \%)$ \\
\hline $\begin{array}{l}\text { Buttocks, perineum, and/or } \\
\text { genitals }\end{array}$ & $5(14 \%)$ & $2(6 \%)$ \\
\hline
\end{tabular}

Number of anatomical regions affected
Table 1 Continued

\begin{tabular}{|cll|}
\hline Variable & $\begin{array}{l}\text { Intervention } \\
\mathbf{n = 3 7}\end{array}$ & $\begin{array}{l}\text { Control } \\
\mathbf{n}=\mathbf{3 5}\end{array}$ \\
\hline 1 & $24(65 \%)$ & $21(60 \%)$ \\
\hline 2 & $8(22 \%)$ & $9(26 \%)$ \\
3 & $5(14 \%)$ & $4(11 \%)$ \\
4 & $0(0 \%)$ & $1(3 \%)$ \\
\hline
\end{tabular}

Required medication in the ED

$\begin{array}{lll}\text { Paracetamol } & 32(86 \%) & 33(94 \%) \\ \text { Ibuprofen } & 26(70 \%) & 28(80 \%) \\ \text { Oxycodone } & 21(57 \%) & 21(60 \%) \\ \text { Fentanyl } & 28(76 \%) & 27(77 \%) \\ \text { Nitrous } & 4(11 \%) & 4(11 \%) \\ \text { Ketamine } & 1(3 \%) & 1(3 \%) \\ \text { Methoxyflurane } & 2(5 \%) & 1(3 \%) \\ \text { Morphine } & 1(3 \%) & 0(0 \%) \\ \text { Midazolam } & 1(3 \%) & 0(0 \%)\end{array}$

Polypharmacy

$\begin{array}{lll}0 & 1(3 \%) & 0(0 \%) \\ 1 & 4(11 \%) & 3(9 \%) \\ 2 & 4(11 \%) & 4(11 \%) \\ 3 & 14(38 \%) & 12(34 \%) \\ 4 & 10(27 \%) & 12(34 \%) \\ 5 & 2(5 \%) & 4(11 \%) \\ 6 & 2(5 \%) & 0(0 \%)\end{array}$

Distraction techniques

\begin{tabular}{|c|c|c|}
\hline Nil & $13(35 \%)$ & $9(26 \%)$ \\
\hline Lollies/food & $1(3 \%)$ & $4(11 \%)$ \\
\hline Sleeping & $2(5 \%)$ & $1(3 \%)$ \\
\hline $\begin{array}{l}\text { Television/phone } \\
\text { distraction }\end{array}$ & $15(41 \%)$ & $11(31 \%)$ \\
\hline Bubbles/toys & $5(14 \%)$ & $7(20 \%)$ \\
\hline $\begin{array}{l}\text { Music therapy/clown } \\
\text { doctors }\end{array}$ & $1(3 \%)$ & $2(6 \%)$ \\
\hline Ditto distraction device & $0(0 \%)$ & $1(3 \%)$ \\
\hline \multicolumn{3}{|c|}{ Definitive dressings applied in ED } \\
\hline $\begin{array}{l}\text { Acticoat 3+Mepitel + } \\
\text { Hypafix }\end{array}$ & $13(35 \%)$ & $10(29 \%)$ \\
\hline $\begin{array}{l}\text { Acticoat } 7+\text { Mepitel + } \\
\text { Hypafix }\end{array}$ & $7(19 \%)$ & $8(23 \%)$ \\
\hline Mepilex Ag+Hypafix & $16(43 \%)$ & $16(46 \%)$ \\
\hline Paraffin wax & $1(3 \%)$ & $1(3 \%)$ \\
\hline \multirow{2}{*}{$\begin{array}{l}\text { Time (minutes) to ED } \\
\text { presentation }\end{array}$} & $n=36$ & $\mathrm{n}=34$ \\
\hline & $90(66-137)$ & $79(60-119)$ \\
\hline Time (minutes) spent in ED & $106.5(66-151)$ & $113(76-180)$ \\
\hline $\begin{array}{l}\text { Time (minutes) dressing } \\
\text { was applied to burn }\end{array}$ & $34(22-61)$ & $35(5-150)$ \\
\hline
\end{tabular}

Continued 


\begin{tabular}{lll}
\hline Table 1 Continued & Intervention & $\begin{array}{l}\text { Control } \\
\mathbf{n = 3 5}\end{array}$ \\
\hline Variable & $36(97 \%)$ & $34(97 \%)$ \\
\hline $\begin{array}{l}\text { Documented first aid } \\
\text { (20 min CRW) }\end{array}$ & $11(30 \%)$ & $7(20 \%)$ \\
QAS applied Burnaid & $8(22 \%)$ & $11(31 \%)$ \\
QAS applied PVC film & $8(22 \%)$ & $9(26 \%)$ \\
\hline High pain risk stratum &
\end{tabular}

Data are presented as median (IQR) for continuous measures, and $\mathrm{N}(\%)$ for categorical measures unless stated otherwise.

${ }^{*}$ As a result of patients having multiple burns to different anatomical regions, LDI scans were taken of 91 burn wounds from 58 patients: $n=48$ burns for the intervention group and $n=43$ wounds for the control.

CRW, cold running water; ED, emergency department; LDI, laser Doppler imaging; n, number of participants; PU, perfusion units; PVC, plasticised polyvinylchloride; QAS, Queensland ambulance service; TBSA, total body surface area.

application (mean difference: $-0.1,95 \% \mathrm{CI}-0.7$ to 0.5 , $\mathrm{p}=0.72$ ), predefinitive dressing application (mean difference: $-0.3,95 \% \mathrm{CI}-1$ to $0.5, \mathrm{p}=0.51$ ), or postdefinitive dressing application (mean difference: $0,95 \% \mathrm{CI}-0.8$ to $0.9, \mathrm{p}=0.92)$.

\section{Ancillary pain measures}

\section{Parent and caregiver pain scores (observer VAS)}

There were no significant differences in pain scores between the control and intervention group for observational pain ratings from parents and caregivers assessed using the VAS Observer in the ED. No significant betweengroup differences in VAS Observer pain scores were found between the intervention and control groups for postrandomised dressing application (mean difference: $1,95 \% \mathrm{CI}-8$ to $11, \mathrm{p}=0.78)$, predefinitive dressing application (mean difference: $0,95 \% \mathrm{CI}-11$ to $11, \mathrm{p}=0.96$ ), or postdefinitive dressing application (mean difference: 6 , $95 \%$ CI -7 to $18, \mathrm{p}=0.36$ ) time points.

\section{Child reported pain (FPS-R and VAS)}

Child self-report pain scores measured using the FPS-R and VAS showed no significant between-group differences. Self-report FPS-R scores assessed postdressing application (mean difference: $0.3,95 \%$ CI -1.7 to 2.2 , $\mathrm{p}=0.78$ ), predefinitive application (mean difference: 0.6 , $95 \% \mathrm{CI}-1.8$ to $2.9, \mathrm{p}=0.64$ ), and postdefinitive dressing application (mean difference: $0.1,95 \%$ CI -3.1 to 3.3 , $\mathrm{p}=0.96$ ) showed no significant group differences. As burn injuries often affect infants and children under the age of 5 , a small number of children recruited into the trial were aged over eight. The VAS for pain is designed for children aged 8 years and older. As a consequence of the median patient age, low numbers of participants were able to use this self-report pain scale and therefore limited statistical

Table 2 Acute pain scores in the ED

\begin{tabular}{|c|c|c|c|c|c|c|c|c|}
\hline Pain scale & Time point & $\mathbf{N}$ & $\begin{array}{l}\text { Intervention } \\
\text { Mean (SD) }\end{array}$ & $\mathbf{N}$ & $\begin{array}{l}\text { Control } \\
\text { Mean (SD) }\end{array}$ & *Adjusted mean difference & $95 \% \mathrm{Cl}$ & $P$ value \\
\hline \multirow{5}{*}{$\begin{array}{l}\text { FLACC } \\
(0-10 \text { scale) }\end{array}$} & T1 & 35 & $1.2(2.1)$ & 23 & $0.7(1.4)$ & - & - & - \\
\hline & T2 & 36 & $0.4(1.0)$ & 35 & $0.4(0.7)$ & -0.1 & -0.7 to 0.5 & 0.72 \\
\hline & T3 & 36 & $0.4(1.2)$ & 34 & $0.6(1.6)$ & -0.3 & -1 to 0.5 & 0.51 \\
\hline & $\mathrm{T} 4$ & 35 & $0.8(1.7)$ & 33 & $0.7(1.5)$ & 0 & -0.8 to 0.9 & 0.92 \\
\hline & Peak pain & 36 & $3.4(2.4)$ & 34 & $3.9(2.8)$ & 0.6 & 1.7 to 0.5 & 0.29 \\
\hline \multirow{4}{*}{$\begin{array}{l}\text { VAS } \\
(0-100)\end{array}$} & $\mathrm{T} 1$ & 9 & $38(29)$ & 2 & $20(14)$ & - & - & - \\
\hline & T2 & 10 & $20(22)$ & 4 & $28(36)$ & -14 & -37 to 9 & 0.22 \\
\hline & T3 & 11 & $16(21)$ & 5 & $8(18)$ & 4 & -18 to 26 & 0.74 \\
\hline & T4 & 7 & $31(25)$ & 4 & $25(44)$ & -1 & -31 to 29 & 0.96 \\
\hline \multirow{4}{*}{$\begin{array}{l}\text { FPS-R } \\
(0-10)\end{array}$} & $\mathrm{T} 1$ & 9 & $3.3(3.7)$ & 7 & $3.6(2.6)$ & - & - & - \\
\hline & T2 & 10 & $2.8(4.2)$ & 8 & $2.4(3.0)$ & 0.3 & -1.7 to 2.2 & 0.78 \\
\hline & T3 & 11 & $1.5(3.3)$ & 11 & $1.3(3.1)$ & 0.6 & -1.8 to 2.9 & 0.64 \\
\hline & T4 & 10 & 2.9 (3.5) & 10 & $3.0(4.1)$ & 0.1 & -3.1 to 3.3 & 0.96 \\
\hline \multirow{4}{*}{$\begin{array}{l}\text { VAS Observer } \\
(0-100)\end{array}$} & $\mathrm{T} 1$ & 34 & $32(28)$ & 22 & 30 (21) & - & - & - \\
\hline & T2 & 34 & $22(24)$ & 31 & $21(19)$ & 1 & -8 to 11 & 0.78 \\
\hline & T3 & 35 & $18(20)$ & 34 & $18(25)$ & 0 & -11 to 11 & 0.96 \\
\hline & $\mathrm{T} 4$ & 33 & $24(25)$ & 32 & $18(26)$ & 6 & -7 to 18 & 0.36 \\
\hline
\end{tabular}

${ }^{*}$ Adjusted mean difference=intervention group - control group.

FLACC, face, legs, activity, cry, consolability; FPS-R, faces pain scale revised; T1, prerandomised dressing application; T2, postrandomised dressing application; T3, predefinitive dressing application; T4, postdefinitive dressing application; VAS, Visual Analogue Scale. 
Table 3 Physiological measures in the ED

\begin{tabular}{|c|c|c|c|c|c|c|c|c|}
\hline Measure & Time point & $\mathbf{N}$ & $\begin{array}{l}\text { Intervention } \\
\text { Mean (SD) }\end{array}$ & $\mathbf{N}$ & $\begin{array}{l}\text { Control } \\
\text { Mean (SD) }\end{array}$ & ${ }^{\star}$ Adjusted mean difference & $95 \% \mathrm{Cl}$ & $P$ value \\
\hline \multirow{3}{*}{$\begin{array}{l}\text { Pulse } \\
\text { (beats/min) }\end{array}$} & $\mathrm{T} 1$ & 34 & $111(27)$ & 24 & $112(20)$ & - & - & - \\
\hline & T3 & 33 & $105(26)$ & 32 & $113(21)$ & -8 & -16 to 1 & 0.07 \\
\hline & T4 & 29 & $109(25)$ & 31 & $113(24)$ & -3 & -12 to 6 & 0.52 \\
\hline \multirow{2}{*}{$\begin{array}{l}\text { Temperature } \\
\left({ }^{\circ} \mathrm{C}\right)\end{array}$} & T3 & 36 & 36.43 & 33 & 36.33 & 0.12 & -0.12 to 0.37 & 0.33 \\
\hline & $\mathrm{T} 4$ & 34 & 36.44 & 33 & 36.32 & 0.14 & -0.14 to 0.40 & 0.29 \\
\hline \multirow{2}{*}{$\begin{array}{l}\text { Alpha-amylase } \\
\text { (units/mL) }\end{array}$} & & & Mean $(\times / S D) \dagger$ & & Mean $(x / S D) \dagger$ & Ratio of means $†$ & $95 \% \mathrm{Cl}$ & \\
\hline & $\mathrm{T} 1$ & 19 & $48(\times / 2)$ & 8 & $46(\times / 3)$ & - & - & - \\
\hline
\end{tabular}

${ }^{*}$ Adjusted mean difference $=$ intervention group - control group.

†Alpha-amylase data reported as geometric mean, geometric SD, and ratio of geometric means.

mL, millilitre; T1, prerandomised dressing application; T2, postrandomised dressing application; T3, predefinitive dressing application; T4, postdefinitive dressing application.

tests that could be performed. Median self-report VAS scores are presented in table 2 but should be interpreted with consideration of this sample size limitation.

\section{Secondary outcomes}

Physiological measures

No significant difference in mean pulse rate (mean difference: $-3,95 \%$ CI -11 to 5 , $\mathrm{p}=0.41$ ) or temperature (mean difference: $0.6,95 \% \mathrm{CI}-0.13$ to $0.24, \mathrm{p}=0.53$ ) was detected between intervention and control groups following the application of the randomised dressings in the ED (see table 3).

\section{Re-epithelialisation}

Median (IQR) time to re-epithelialisation for the intervention group was 9 days (6.25-10.75) and 9 days (7.5-14) for the control group. Clinical assessment from treating surgeons showed no significant between-group differences in time to $95 \%$ re-epithelialisation, with a median difference $(95 \% \mathrm{CI}$ ) equal to -1 ( -3 to 1$), \mathrm{p}=0.26$. With regards to the blinded assessment of burn wound images, exact agreement between the treating surgical consultants and blinded review panel was used to examine agreement between health professionals measuring time to re-epithelialisation. ${ }^{40}$ Agreement on evaluation of re-epithelialisation was found to be good (69\% agreement) between the three expert reviewers and the treating surgeons (see online supplemental appendix 1 for additional agreement data).

\section{Biochemical stress markers}

No significant difference in sAA was found between the intervention and control group following the application of the randomised dressing during acute care in the ED (see table 3). Children who received Burnaid dressings did not show a reduction in the biochemical stress marker in comparison to paediatric patients who received PVC film (geometric mean ratio: 1.53 , 95\% CI 0.93 to $2.53, p=0.10)$. Levels of sAA collected in the waiting room during dressing changes one (geometric mean ratio: 1 , $95 \%$ CI 0.65 to $1.56, p=0.97$ ) and two (geometric mean ratio: $1.14,95 \%$ CI 0.48 to $2.71, \mathrm{p}=0.75$ ) showed no significant differences between children who received Burnaid dressings in the ED and those who received PVC film (see online supplemental appendix 2).

Pain at first, second and third dressing changes

Pain scores assessed in the Burns Outpatient Department during follow-up dressing changes one to three are reported in online supplemental appendix 3 for the two treatment groups. No statistical differences in observational or child self-report follow-up pain scores were found between children who received Burnaid dressings and those who received PVC film during acute care. Temperature and pulse rate assessed during follow-up dressing changes (as physiological indicators of pain) also showed no significant group differences over dressing changes one to three (see online supplemental appendix 2 for physiological data).

\section{Staff and caregiver perspectives on dressings}

Dressing satisfaction from clinical staff, in addition to parents and caregivers, assessed in the ED during acute care is presented in online supplemental appendix 4 . No significant differences in ease of dressing application, removal, flexibility or conformity were identified between the two groups from ED nursing staff. Parents are caregivers reported higher satisfaction scores for ease of dressing application for children who received Burnaid dressings, in comparison to those who received PVC film $(\mathrm{p}=0.013)$. Parent $/$ caregiver satisfaction scores were also 
higher for ease of dressing removal within the Burnaid arm, in comparison to the control arm $(p=0.045)$. Furthermore, parents and caregivers reported higher satisfaction scores for ease of movement for children who received Burnaid, in comparison to paediatric patients who received PVC film in the ED $(p=0.047)$. Last, no significant differences in perceived patient comfort were identified between the two groups from parents and caregivers using the $0-10$ NRS.

\section{DISCUSSION}

There has been an emergence of research demonstrating the importance of acute pain control in traumatic injuries, emphasising the association between untreated pain and maladaptive outcomes such as: prolonged wound healing, ${ }^{45}$ long-term emotional disorders, ${ }^{67}$ and chronic pain conditions. ${ }^{89}$ Pain is a chief problem for patients with burn injuries in the acute setting. ${ }^{41}{ }^{42}$ Therefore, prehospital and acute care providers have a crucial role in recognising and reducing the burden of pain for these patients. Reducing acute pain is of particular importance for paediatric burn patients who often have to undergo numerous painful and distressing medical procedures during their care. The better pain and distress are managed during a child's first visit to the ED for burn wound treatmentthe lower the child's chances are of developing anticipatory anxiety and avoidance behaviours for future medical procedures. ${ }^{43}$ Effective non-pharmacological interventions for the management of acute burn pain are needed to supplement pharmacological methods of pain reduction in paediatric patients. ${ }^{364}$ We were pleasantly reassured to find most burn patients presenting to our ED had mild to no pain. Because of this, examining the effectiveness of acute burn dressings on reducing acute pain score was restricted-and results from this prospective RCT should be interpreted with the acknowledgement of this limitation. At present, there are no high level trials supporting the use of Burnaid hydrogel dressings for acute burn management. The aim of this trial was to fill this gap in the literature, and examine the effectiveness of Burnaid dressings on reducing acute pain scores in children with thermal burns. To the best of our knowledge, this is the first prospective RCT conducted in a paediatric burn population examining the analgesic properties of a hydrogel burn dressing in an ED setting.

Results from this prospective RCT should be interpreted with consideration of several limitations. First, very few participants had moderate to severe pain scores following their initial presentation to the QCH prior to recruitment into the trial-see online supplemental appendix 5 for complete pain score frequencies. More than $60 \%$ of paediatric burn patients received observational pain scores of zero (out of 10 using the FLACC pain scale) from ED nursing staff. Moreover, an additional 19\% of children received pain ratings equal to one (using the 10-point scale) following initial presentation to the ED. A significant effect of the intervention on reducing acute burn pain might not have been identified in this trial because pain scores were so low following patient's first presentation to hospital for their burn. Second, prehospital and referral services in Queensland acted to provide comprehensive pharmacotherapies for pain management to paediatric patients with thermal burns during transportation to the QCH. So much so that pain scores might have been too low to observe a significant reduction following application of the intervention or control. A large proportion $(78 \%)$ of patients enrolled in the trial received three or more medication classes during their acute burn care-the most common combination being paracetamol, ibuprofen, fentanyl for both groups (see table 1).

The third limitation also relates to prehospital care, and includes the use of different acute burn dressings during patient transport to hospital, prior to randomisation and enrolment in the trial. As this was a pragmatic trial aiming to simulate real-world clinical scenarios within the $\mathrm{ED}$, the application of prehospital acute burn dressings was not an exclusion criterion for participation. However, this meant that some participants received PVC film or Burnaid prior to presenting to the QCH, which may have had confounding effects. Furthermore, 16 participants ( $n=4$ intervention and $n=12$ control) did not keep their randomised dressings on for the required $20 \mathrm{~min}$ duration. Two main factors challenged dressing adherence during acute data collection in the ED-excessive wound exudate beneath the PVC film causing the dressings to slip off participant's burns, and a number of paediatric patients pulling at and removing their own dressings. Fidelity in these instances was compromised, and is a limitation of the current trial. Fourth, where paediatric burn patients received their first aid cooling (ie, on-scene with paramedics, at home in the shower, or within the ED) was not delineated in the dataset-and this is acknowledged as a significant limitation. In addition, while all administered analgesia was documented for participants, where this analgesia was administered was also not delineated in the dataset. This is further acknowledged as a significant research limitation.

The last limitation relates to potential moderating effects. Non-pharmacological interventions such as distraction are commonplace during paediatric medical procedures. Almost $70 \%$ of all participants received additional distraction techniques during their acute burn treatment in the ED such as video distraction using mobile phones and television, clown doctors, music therapists, bubbles, toys and lollies (see table 1). These nonpharmacological interventions were also left in place to simulate a real-world pragmatic trial, however could have moderated the effect of the intervention. An effect of the intervention on reducing acute pain scores might not have been detected due to the low pain scored at initial presentation, analgesia on-board at the time of recruitment, or other confounding factors such as the application of prehospital burn dressings prior to enrolment in the trial. It is therefore recommended that this research 
be replicated in the prehospital setting-where acute pain scores are anticipated to be higher and the application of prehospital burn dressings and analgesia can be better controlled.

\section{CONCLUSION}

It was predicted that Burnaid dressings would provide superior analgesia for paediatric burn patients when applied as an adjunct to CRW first aid, in comparison to PVC film (current standard practice). However, the effect of the intervention on reducing acute pain scores was not supported in this investigation and we were unable to show a clinically relevant treatment effect caused by the intervention-Burnaid hydrogel dressings. Results from this RCT found no significant between-group differences in observational pain scores assessed using the FLACC pain scale from ED nursing staff-the primary outcome of the trial. Moreover, no significant group differences in parent/caregiver pain scores or child self-report pain scores were identified during acute care in the ED or follow-up wound care in the Burns OPD. The effect of the intervention on additional outcomes including, time to re-epithelialisation, stress, temperature, heart rate and need for analgesic medication was also not supported. Ease of dressing application and removal, in addition to ease of patient movement while dressings were applied, were higher for the Burnaid group in accordance with parent and caregiver ratings. Dressing satisfaction measures from clinical staff within the ED found no significant differences between patients who received Burnaid and those who received PVC film. Moreover, no difference in perceived comfort ratings from parents and caregivers were identified between the two groups.

As aforementioned, results from this prospective trial should be interpreted with consideration of several limitations including low pain scores following initial patient presentation, analgesia on-board at the time of recruitment, and pragmatic issues with dressing compliance. Additional research is still required to examine the effectiveness of different acute burn dressings as analgesic adjuncts to running water first aid. Research investigating adjunctive methods of pain control for children with burns holds great translational value. It was predicted that an acute burn dressing with additional cooling and evaporative properties would provide superior pain relief for children with thermal burns, in comparison to PVC film. This was not supported, and Burnaid dressings do not appear to provide superior pain relief in comparison to PVC film when applied as an acute burn dressing following first aid and initial presentation to the ED.

\footnotetext{
Author affiliations

${ }^{1}$ Centre for Children's Burns and Trauma Research, Centre for Children's Health Research, South Brisbane, Queensland, Australia

${ }^{2}$ Pegg Leditschke Paediatric Burns Centre, The Queensland Children's Hospital, Herston, Queensland, Australia

${ }^{3}$ Faculty of Medicine, The University of Queensland, Brisbane, Queensland, Australia
}

${ }^{4}$ Faculty of Health, School of Nursing, Queensland University of Technology, Brisbane, Queensland, Australia

${ }^{5}$ Institute of Health and Biomedical Innovation, Queensland University of Technology, Brisbane, Queensland, Australia

Acknowledgements The authors want to thank all the children and families for their time and participation in our research. They also wish to acknowledge and give thanks to the Burns, Surgical, and ED staff at the Queensland Children's Hospital for their support in the development and implementation of this research.

Contributors RMK and BRG conceived the research, designed the trial and obtained research funding. MDH undertook participant recruitment, acute and follow-up data collection, data management, and interpretation of results. MC provided statistical support and conducted the formal analyses. MDH wrote the draft manuscript, and all authors provided critical review of the article and approved the final manuscript. MDH takes responsibility for the paper as a whole.

Funding This work was supported by a research grant given to The University of Queensland by Mundipharma (Grant Number: 364010399) and an Australian Government Research Training Scholarship awarded to the primary investigator. Mundipharma had no role in the development of the trial, data collection, data analysis, or interpretation of results.

Competing interests All authors who contributed to this original research manuscript declare no conflicts of interests. All authors declare no financial or other interests in the product (Burnaid) or distributor of the product (Mundipharma). All authors declare no past or existing relationships with the manufacturer or distributor of the product. Moreover, all authors declare no additional associations with the product manufacturer or distributor including consultancies, stock ownership, or other equity interests or patent-licensing arrangements.

Patient consent for publication Not required.

Ethics approval This trial received ethics approval from The Queensland Children's Health Service Human Research Ethics Committee (approval number: HREC/16/QRCH/322) and The University of Queensland Ethics Committee (clearance number: 2017000979).

Provenance and peer review Not commissioned; externally peer reviewed.

Data availability statement Data are available upon reasonable request. Additional data available upon request.

Supplemental material This content has been supplied by the author(s). It has not been vetted by BMJ Publishing Group Limited (BMJ) and may not have been peer-reviewed. Any opinions or recommendations discussed are solely those of the author(s) and are not endorsed by BMJ. BMJ disclaims all liability and responsibility arising from any reliance placed on the content. Where the content includes any translated material, BMJ does not warrant the accuracy and reliability of the translations (including but not limited to local regulations, clinical guidelines, terminology, drug names and drug dosages), and is not responsible for any error and/or omissions arising from translation and adaptation or otherwise.

Open access This is an open access article distributed in accordance with the Creative Commons Attribution Non Commercial (CC BY-NC 4.0) license, which permits others to distribute, remix, adapt, build upon this work non-commercially, and license their derivative works on different terms, provided the original work is properly cited, appropriate credit is given, any changes made indicated, and the use is non-commercial. See: http://creativecommons.org/licenses/by-nc/4.0/.

ORCID iD

Maleea Denise Holbert http://orcid.org/0000-0002-9902-5122

\section{REFERENCES}

1 Scott LE, Crilly J, Chaboyer W, et al. Paediatric pain assessment and management in the emergency setting: the impact of a paediatric pain bundle. Int Emerg Nurs 2013;21:173-9.

2 Summer GJ, Puntillo KA, Miaskowski C, et al. Burn injury pain: the continuing challenge. J Pain 2007;8:533-48.

3 Morgan M, Deuis JR, Frøsig-Jørgensen M, et al. Burn pain: a systematic and critical review of epidemiology, pathophysiology, and treatment. Pain Med 2018;19:708-34.

4 Brown NJ, Kimble RM, Rodger S, et al. Play and heal: randomized controlled trial of Ditto ${ }^{\mathrm{TM}}$ intervention efficacy on improving reepithelialization in pediatric burns. Burns 2014;40:204-13. 
5 Brown NJ, Kimble RM, Gramotnev G, et al. Predictors of reepithelialization in pediatric burn. Burns 2014;40:751-8.

6 De Young AC, Kenardy JA, Cobham VE, et al. Prevalence, comorbidity and course of trauma reactions in young burn-injured children. J Child Psychol Psychiatry 2012;53:56-63.

7 Norman SB, Stein MB, Dimsdale JE, et al. Pain in the aftermath of trauma is a risk factor for post-traumatic stress disorder. Psychol Med 2008;38:533-42.

8 Dauber A, Osgood PF, Breslau AJ, et al. Chronic persistent pain after severe burns: a survey of 358 burn survivors. Pain Med 2002;3:6-17.

9 Wollgarten-Hadamek I, Hohmeister J, Zohsel K, et al. Do schoolaged children with burn injuries during infancy show stress-induced activation of pain inhibitory mechanisms? Eur J Pain 2011;15:423. e1-e10.

10 Cuttle L, Kempf M, Liu P-Y, et al. The optimal duration and delay of first aid treatment for deep partial thickness burn injuries. Burns 2010;36:673-9.

11 Bartlett N, Yuan J, Holland AJA, et al. Optimal duration of cooling for an acute scald contact burn injury in a porcine model. J Burn Care Res 2008;29:828-34.

12 The Australian and New Zealand Burn Association. Emergency management of severe burns. $13 \mathrm{ed}$. Australian and New Zealand: Australian and New Zealand Burn Association, 2009.

13 Cuttle L, Kempf M, Kravchuk O, et al. The optimal temperature of first aid treatment for partial thickness burn injuries. Wound Repair Regen 2008;16:626-34

14 Griffin BR, Frear CC, Babl F, et al. Cool running water first aid decreases skin grafting requirements in pediatric burns: a cohort study of two thousand four hundred ninety-five children. Ann Emerg Med 2020;75:75-85.

15 Allison K, Porter K. Consensus on the pre-hospital approach to burns patient management. Injury 2004;35:734-8.

16 Fein M, Quinn J, Watt K, et al. Prehospital paediatric burn care: new priorities in paramedic reporting. Emerg Med Australas 2014:26:609-15.

17 Cuttle L, Kempf M, Kravchuk O, et al. The efficacy of Aloe vera, tea tree oil and saliva as first aid treatment for partial thickness burn injuries. Burns 2008;34:1176-82.

18 Holbert MD, Griffin BR, McPhail SM, et al. Effectiveness of a hydrogel dressing as an analgesic adjunct to first aid for the treatment of acute paediatric thermal burn injuries: study protocol for a randomised controlled trial. Trials 2019;20:13.

19 Milner RH, Hudson SJ, Reid CA. Plasticized polyvinyl chloride film as a primary burns dressing: a microbiological study. Burns Incl Therm Inj 1988; 14:62-5.

20 Lendrum J, Bowen-Jones E. A new dressing for burns: Enclosure in a plasticized polyvinyl chloride sheet. Burns 1976;2:86-9.

21 Merkel S, Voepel-Lewis T, Malviya S. Pain assessment in infants and young children: the FLACC scale: a behavioral tool to measure pain in young children. Am J Nurs 2002;102:55-8.

22 Willis MHW, Merkel SI, Voepel-Lewis T, et al. FLACC behavioral pain assessment scale: a comparison with the child's self-report. Pediatr Nurs 2003;29:195-8.

23 Gomez R, Barrowman N, Elia S. Reliability of the flacc scale for evaluating pain in toddlers. Irish J Med Sci 2012;181:456.
24 von Baeyer CL. Children's self-report of pain intensity: what we know, where we are headed. Pain Res Manag 2009;14:39-45.

25 Hicks CL, von Baeyer CL, Spafford PA, et al. The faces pain scalerevised: toward a common metric in pediatric pain measurement. Pain 2001;93:173-83.

26 Bailey B, Gravel J, Daoust R. Reliability of the visual analog scale in children with acute pain in the emergency department. Pain 2012;153:839-42.

27 Stinson JN, Kavanagh T, Yamada J, et al. Systematic review of the psychometric properties, Interpretability and feasibility of self-report pain intensity measures for use in clinical trials in children and adolescents. Pain 2006;125:143-57.

28 Taddio A, O'Brien L, Ipp M, et al. Reliability and validity of observer ratings of pain using the visual analog scale (vas) in infants undergoing immunization injections. Pain 2009;147:141-6.

29 Brown NJ, Kimble RM, Rodger S, et al. Biological markers of stress in pediatric acute burn injury. Burns 2014;40:887-95.

30 Dibildox M, Jeschke MG, Herndon DN. Burn injury, rule of nines. In: Vincent J-L, Hall JB, eds. Encyclopedia of intensive care medicine. Berlin, Heidelberg: Springer Berlin Heidelberg, 2012: 417-9.

31 Wang X-Q, Mill J, Kravchuk O, et al. Ultrasound assessed thickness of burn scars in association with laser Doppler imaging determined depth of burns in paediatric patients. Burns 2010;36:1254-62.

32 Mill J, Cuttle L, Harkin DG, et al. Laser Doppler imaging in a paediatric burns population. Burns 2009;35:824-31.

33 Gill P. The critical evaluation of laser Doppler imaging in determining burn depth. Int J Burns Trauma 2013;3:72-7.

34 Štětinský J, Klosová $\mathrm{H}$, Kolářová $\mathrm{H}$, et al. The time factor in the LDI (laser Doppler imaging) diagnosis of burns. Lasers Surg Med 2015;47:196-202.

35 Wearn C, Lee KC, Hardwicke J, et al. Prospective comparative evaluation study of laser Doppler imaging and thermal imaging in the assessment of burn depth. Burns 2018;44:124-33.

36 Miller K, Rodger S, Kipping B, et al. A novel technology approach to pain management in children with burns: a prospective randomized controlled trial. Burns 2011;37:395-405.

37 StataCorp. StataCorp. 2019. Stata Statistical Software: Release 16. College Station, TX: StataCorp LLC..

38 Limpert E, Stahel WA. Problems with using the normal distribution-and ways to improve quality and efficiency of data analysis. PLoS One 2011;6:e21403.

39 Chester SJ, Tyack Z, De Young A, et al. Efficacy of hypnosis on pain, wound-healing, anxiety, and stress in children with acute burn injuries: a randomized controlled trial. Pain 2018;159:1790.

40 de Vet HCW, Terwee CB, Knol DL, et al. When to use agreement versus reliability measures. J Clin Epidemiol 2006;59:1033-9.

41 Yuxiang L, Lingjun Z, Lu T, et al. Burn patients' experience of pain management: a qualitative study. Burns 2012;38:180-6.

42 Nedelec B, Carrougher GJ. Pain and pruritus Postburn injury. J Burn Care Res 2017:38:142-5.

43 Michalska KJ, Feldman JS, Abend R, et al. Anticipatory effects on perceived pain: associations with development and anxiety. Psychosom Med 2018;80:853-60.

44 Miller K, Rodger S, Bucolo S, et al. Multi-modal distraction. Using technology to combat pain in young children with burn injuries. Burns 2010;36:647-58. 